Preprint typeset in JHEP style - HYPER VERSION

TAUP - 2839/06

hep-ph/0610084

February 8, 2020

\title{
The BFKL Pomeron Calculus in zero transverse dimensions: diffractive processes and survival probability for central diffractive production
}

\author{
M. Kozlov ${ }^{a)} *$, E. Levin ${ }^{a)} \dagger$, V. Khachatryan ${ }^{b)} \ddagger$ and J. Miller ${ }^{a)} \S$ \\ a) Department of Particle Physics, School of Physics and Astronomy \\ Raymond and Beverly Sackler Faculty of Exact Science \\ Tel Aviv University, Tel Aviv, 69978, Israel \\ b) Theory Division, Yerevan Physics Institute, Yerevan, 375036, Armenia
}

ABSTRACT: In this paper we discuss the processes of diffractive production in the framework of the BFKL Pomeron calculus in zero transverse dimension. Considering the diffractive production of a bunch of particles with not very large masses, namely, $\ln \left(M^{2} / m^{2}\right) \ll \frac{1}{\bar{\alpha}_{S}} \ln \left(\frac{N_{c}^{2}}{\bar{\alpha}_{S}^{2}}\right)$, we found explicit formulae for calculation of the cross sections for the single and double diffractive production as well as for the value of the survival probability for the diffractive central production. These formulae include the influence of the correlations due to so called Pomeron loops on the values of all discussed observables. The comparison with the other approaches on the market is given. The main conclusion of this comparison: the Mueller-PatelSalam-Iancu approximation gives sufficiently good descriptions and close to the exact result for elastic and diffractive cross section but considerable overshoot the value of the survival probability.

KEYwords: BFKL Pomeron, Diffractive cross sections, Mean field approach, Pomeron loops, Exact solution.

PACS: 13.85-t, 13.85.Hd, 11.55.-m, 11.55.Bq

\footnotetext{
*Email: kozlov@post.tau.ac.il;

${ }^{\dagger}$ Email: leving@post.tau.ac.il, levin@mail.desy.de;

${ }^{\ddagger}$ Email:khavladi@yerphi.am;

§Email:jeremymiller@london.com,jeremy@post.tau.ac.il;
} 


\section{Contents}

$\begin{array}{ll}\text { 1. Introduction } & 1\end{array}$

2. Single diffractive production 4

2.1 The mean field approximation.

2.1.1 General approach.

2.1.2 The BFKL Pomeron calculus in zero transverse dimension. 6

2.2 Diffractive production beyond the mean field approximation 8

3. Double diffractive production $\quad 11$

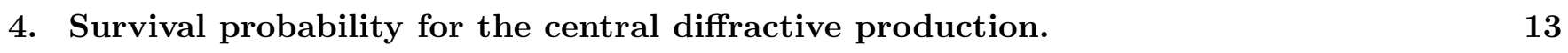

5. Comparison with the approximate approaches. 16

5.1 Our approach versus the mean field approximation. 16

5.2 Our approach versus Mueller - Patel - Salam - Iancu (MPSI) approximation. 17

6. Conclusions 20

\section{Introduction}

Diffractive production processes, being pure quantum mechanical by their origin, have always been a source of interesting information and sometimes even of confusion for all theoretical approaches to high energy scattering. It is enough to mention that due to the AGK cutting rules [1] the total cross section of the diffractive production is equal to the contribution of the first shadowing correction (with opposite sign) to the total cross section.

The goal of this paper is to discuss these processes in the framework of high density QCD [3, 4, 5]. Since the most simple and elegant approach, that allows us to discuss both the total cross sections and the diffractive production on the same footing, is the AGK cutting rules, we choose the particular version of the theoretical approach to high density QCD: the BFKL Pomeron calculus [3, 国. This approach is based on the BFKL Pomeron [6] and the interactions between the BFKL Pomerons [7, 8, 9, 10], which 
are taken into account in the spirit of the Gribov Reggeon Calculus [11. The BFKL Pomeron calculus can be formulated at least in two beautiful forms: as the functional integral [8] and as the evolution equation for the generating functional (see Refs. 12, 13, 14, 15, 16]) . Both of them reproduce the mean field approximation for the total cross sections (see Refs. [5, 17, 18]) as well as leading to the mean field equations for the diffractive amplitude (see Ref. [19]). In both of them we can include so called Pomeron (see Refs. 22, 23, 15, 24, 25, 26]) loops which were not taken into account in the mean field approximation and which are very essential for the processes of the diffraction production especially in the region of large masses of the diffractive system. However, it is not clear at the moment the interrelation between the BFKL Pomeron calculus and the first attempt to suggest the equations for the diffractive processes in the framework of the approach to high density is more general than the mean field approach (see Ref. [27]).

In this paper we discuss the diffractive production in the simplest version of the BFKL Pomeron calculus, namely, in the toy model in which we neglect the fact that the QCD interaction can change the sizes of interacting dipoles. Such system of colourless dipoles reduces to the BFKL Pomeron calculus in zero transverse dimension. The analytical solution for the generating functional has been found in this model (see Ref. [28] and an earlier attempt to solve this problem in Refs. [16, 29, 30]). Using this solution as well as the AGK cutting rules we can develop the theoretical approach to the diffractive processes. The first attempt to build such an approach has been made in Ref. [36], however it was done in the limited kinematic region.

The paper is organized as follows. In the next section we discuss the single diffractive production in both the mean field approximation and in the approximation that includes the Pomeron loops. The equations that govern this process, are written and the analytical solutions are found. However, we are able to solve the problem only in the restricted range of mass for the diffractive system $(M)$, namely,

$$
\frac{1}{\bar{\alpha}_{S}} \ll Y_{0} \equiv \ln \left(M^{2} / m^{2}\right) \ll \frac{1}{\bar{\alpha}_{S}} \ln \left(\frac{N_{c}^{2}}{\bar{\alpha}_{S}^{2}}\right)
$$

where $m$ is a typical mass of soft interaction (say $m=1 \mathrm{GeV}$ ) and $\alpha_{S}$ is the running QCD coupling $\bar{\alpha}_{S}=\left(N_{c} / \pi\right) \alpha_{S}$. In this region we can neglect the fluctuations in the diffractively produced cascade of partons due to the Pomeron loops contribution [31, 33, 34, 35, 36]. It should be stressed that the Pomeron loops are taken into account for the "elastic" scattering with rapidity

$$
Y-Y_{0}=\ln \left(s / M^{2}\right) \geq \frac{1}{\bar{\alpha}_{S}} \ln \left(\frac{N_{c}^{2}}{\bar{\alpha}_{S}^{2}}\right)
$$

In the third section we consider the double diffractive dissociation in the kinematic region of Eq. (1.1) for both produced masses $\left(M_{1}\right.$ and $\left.M_{2}\right)$ but at very high energy

$$
\ln \left(\frac{s m^{2}}{M_{1}^{2} M_{2}^{2}}\right) \geq \frac{1}{\bar{\alpha}_{S}} \ln \left(\frac{N_{c}^{2}}{\bar{\alpha}_{S}^{2}}\right) .
$$


The fourth section is devoted to calculations of the survival probability for a process with a large rapidity gap (LRG) of the following type

$$
\begin{aligned}
\text { hadron }+ \text { hadron } & \longrightarrow \\
\text { hadron }+\{\text { LRG }\} & + \text { system of fixed mass }(\text { di-jet, Higgs meson }, \ldots)\}+\{\text { LRG }\}+\text { hadron }
\end{aligned}
$$

which we can call a central diffractive process. The value of the survival probability of this process is a hot question since this reaction is one of the best for measuring Higgs meson at the LHC ( see Refs. [37, 38, 39, 40, 41, 42]).

In section five we compare our estimates with other approaches that are on the market and in conclusions we summarize the results.

Our approach in this paper is very close to one that has been developed in Ref. [36]. Therefore, it is worthwhile to stress the points on which we differ:

1. In both papers the range of produced mass is restricted by Eq. (1.1), and, therefore, we can neglect the fluctuations due to the Pomeron loops in produced system. It results in using the mean field approximations ('fan', tree Pomeron diagrams) for the produced system in both papers. However, we did not assume that the total energies restricted $\left(Y-Y_{0} \ll \frac{1}{\bar{\alpha}_{S}} \ln \left(\frac{N_{c}^{2}}{\bar{\alpha}_{S}^{2}}\right)\right)$ as it is done in Ref. [36] to justify the use of Mueller-Patel-Salam-Iancu approach [31, 32, 33]. For region, given by Eq. (1.2), we use the exact solution of the BFKL Pomeron calculus that has been found in Ref. [28];

2. In our approach we explore the Kovchegov-Levin equation [19] (see also Refs. [20, 21] where this equation was generalized in the mean field approximation) for diffractive production in the mean field approximation. Being equivalent to AGK cutting rules in the BFKL Pomeron calculus in zero transverse dimension, it has solid foundation in high density QCD (see Refs. [19, 27]) and allows us to develop a more general formalism in calculating the diffractive observables. In this formalism the solution to Kovchegov-Levin equation for $Y_{0}$ in the kinematic range of Eq. (1.1) plays role of the initial condition for the general equation for the scattering amplitude in the rapidity range of $Y-Y_{0}$;

3. Even in section 5.2 where we apply our approach to the Mueller-Patel-Salam-Iancu range of energies we obtain more general formulae since we do not assume that $Y-Y_{0} \gg Y_{0}$ as in Ref. [36].

One of our motivations to discuss diffractive processes stems from the interesting paper of Y. Hatta, E. Iancu, C. Marquet, G. Soyez and D. N. Triantafyllopoulos [27] in which the diffractive dissociation has been considered in high density QCD. In this paper a generalization of Kovchegov-Levin equation [19] is proposed. We wished to check and to confront these equations with the simplest model that we have at our disposal: the BFKL Pomeron calculus in zero transverse dimension. Indeed, we found two features of the diffractive processes in our approach that support the main ideas of Ref. [27]: (i) a kind of factorization between wee partons (Pomerons) produced by the incoming particle at rapidity $Y_{0}$ and the process of creation of the diffractive system by these partons (Pomerons); and (ii) an impressive difference between the mean field approximation and the exact answer. 


\section{Single diffractive production}

\subsection{The mean field approximation.}

\subsubsection{General approach.}

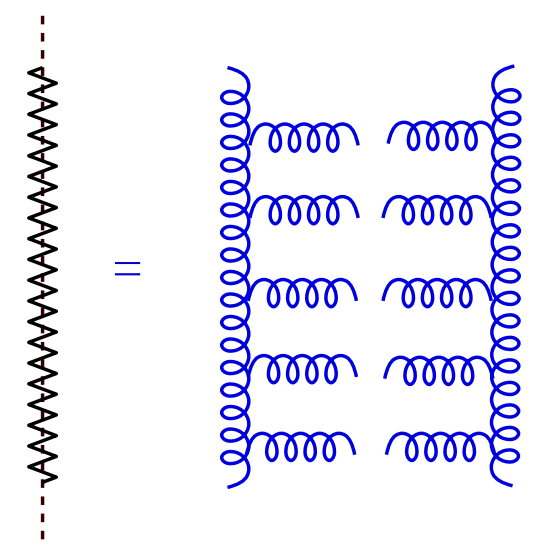

Figure 1: The diagram for the cut BFKL Pomeron that illustrates Eq. (2.1). exchange of the BFKL Pomeron is related to the processes of multi-gluon production in a certain kinematics (see Fig. 1). This equation was proven in Ref. [6]. Using Eq. (2.1), we can express all processes and, in particular, the processes of diffractive production in terms of exchange and interactions of the BFKL Pomerons and/or the cut BFKL Pomerons (see Fig. 2).
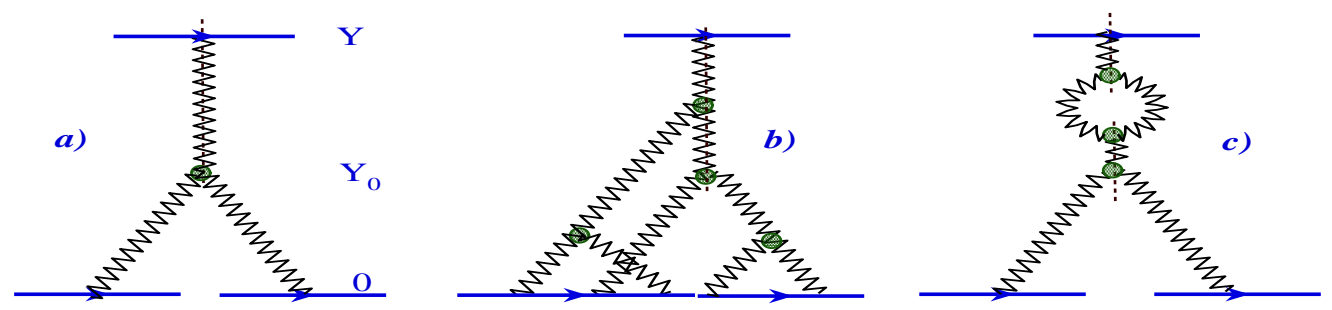

Figure 2: Several examples of the Pomeron diagrams that contribute to the diffractive production of the bunch of gluon with $\ln \left(M^{2} / m^{2}\right)=Y-Y_{0}$ : diagram $a$ ) is the first diagram for the process of the difractive production, while diagram $b$ ) gives an example of more complicated contribution to the diffractive production in the mean field approximation; diagram $c$ ) is the example of the diagrams that we neglected in the mean field approximation as well as in the approach of this paper.

The AGK cutting rules establish the relation between different processes that stem from BFKL Pomeron diagrams. For example, the simple triple Pomeron diagrams in Fig. 3 leads to three inelastic processes: the diffractive production of the system with mass $\ln \left(M^{2} / m^{2}\right)=Y-Y_{0}$ (Fig. 3-A); the multigluon production in the entire kinematic region of rapidity $Y-0$ with the same multiplicity of gluons 
as in one Pomeron ( Fig. 3-B); and the multi-gluon production in the region $Y-0$ but with the same multiplicity of gluons as in one Pomeron only in the rapidity window $Y-Y_{0}$ while for the rapidity $Y_{0}-0$ the gluon multiplicity in two times larger than for one Pomeron( Fig. 3-C). The AGK cutting rules [1] say that the cross sections of these three processes are related as

$$
\sigma_{A}: \sigma_{B}: \sigma_{C}=2:-4: 1
$$

At first sight the cross section of the process B is negative but it should be stressed that one Pomeron also contributes to the same process and the resulting contribution is always positive.

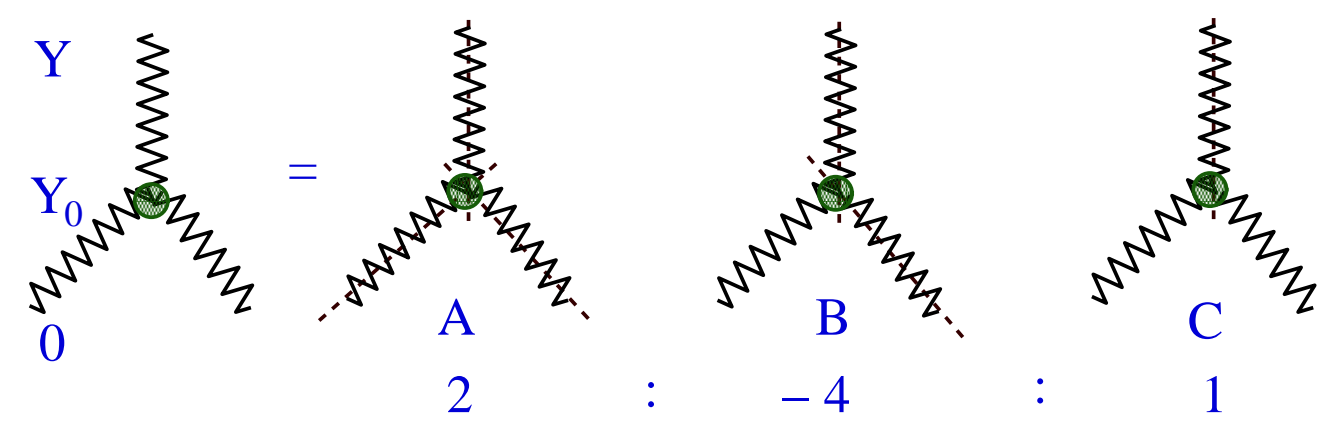

Figure 3: The AGK cutting rules for the triple Pomeron diagram: the diffractive production of the system with mass $\ln \left(M^{2} / m^{2}\right)=Y-Y_{0}$ (Fig. 3-A); the multi-gluon production in the entire kinematic region of rapidity $Y-0$ with the same multiplicity of gluons as in one Pomeron ( Fig. 3-B); and the multi-gluon production in the region $Y-0$ but with the same multiplicity of gluons as in one Pomeron only in the rapidity window $Y-Y_{0}$ while for the rapidity $Y_{0}-0$ the gluon multiplicity in two times larger than for one Pomeron( Fig. 3-C). The zigzag line denotes the Pomeron exchange while the zigzag line crossed y the dotted line stands for the cut Pomeron.

Fig. 1, Fig. 2 and Fig. 3 as well as Eq. (2.2) allow us to understand the equation for the single diffractive production in the mean field approximation that has been written by Kovchegov and Levin [19] and that has the following form (see Fig. 0 for graphical representation of this equation)

$$
\begin{gathered}
\frac{\partial N^{D}\left(\mathbf{x}_{01}, \mathbf{b}, Y, Y_{0}\right)}{\partial Y}=\frac{\alpha C_{F}}{\pi^{2}} \int_{\rho} d^{2} x_{2} \frac{x_{01}^{2}}{x_{02}^{2} x_{12}^{2}} \\
\left(N^{D}\left(\mathbf{x}_{02}, \mathbf{b}+\frac{1}{2} \mathbf{x}_{12}, Y, Y_{0}\right)+N^{D}\left(\mathbf{x}_{12}, \mathbf{b}+\frac{1}{2} \mathbf{x}_{02}, Y, Y_{0}\right)-N^{D}\left(\mathbf{x}_{01}, \mathbf{b}, Y, Y_{0}\right)\right. \\
+N^{D}\left(\mathbf{x}_{02}, \mathbf{b}+\frac{1}{2} \mathbf{x}_{12}, Y, Y_{0}\right) N^{D}\left(\mathbf{x}_{12}, \frac{1}{2} \mathbf{x}_{02}, Y, Y_{0}\right) \\
-4 N^{D}\left(\mathbf{x}_{02}, \mathbf{b}+\frac{1}{2} \mathbf{x}_{12}, Y, Y_{0}\right) N_{0}\left(\mathbf{x}_{12}, \mathbf{b}+\frac{1}{2} \mathbf{x}_{02}, Y\right) \\
\left.+2 N_{0}\left(\mathbf{x}_{02}, \mathbf{b}+\frac{1}{2} \mathbf{x}_{12}, Y\right) N_{0}\left(\mathbf{x}_{12}, \mathbf{b}+\frac{1}{2} \mathbf{x}_{02}, Y\right)\right)
\end{gathered}
$$


A

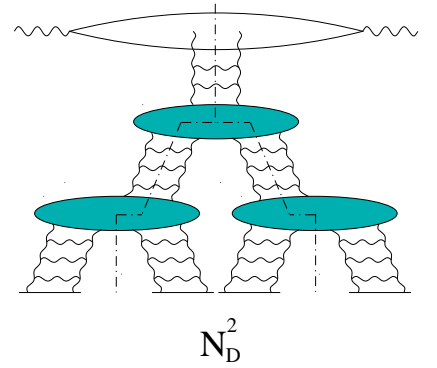

B

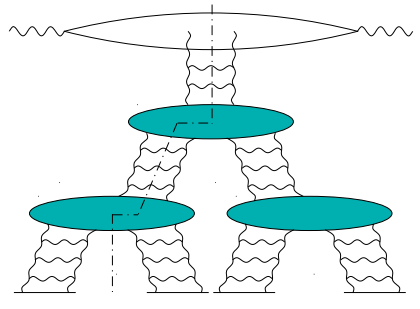

$-4 N_{D} N_{O}$

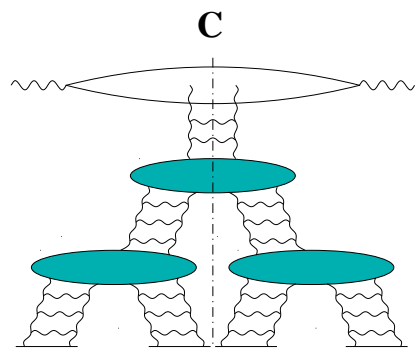

$2 \mathrm{~N}_{\mathrm{O}}^{2}$

Figure 4: Different Pomeron cuts contributing to the cross section of diffractive dissociation which lead to different terms on the right hand side in Eq. (2.3)

The physical meaning of $N_{D}$ is the cross section of the diffractive produced masses $M$ large than $\ln \left(M^{/} m^{2}\right) \geq Y-Y_{0}$ or in other words

$$
N^{D}\left(\mathbf{x}_{01}, \mathbf{b}, Y, Y_{0}\right)=\int_{Y_{0}}^{Y} d Y^{\prime} N^{S D}\left(\mathbf{x}_{01}, \mathbf{b}, Y, Y^{\prime}\right)
$$

where $N^{S D}\left(\mathbf{x}_{01}, \mathbf{b}, Y, Y^{\prime}\right)$ is the cross section of the diffractive production at energy $Y$ with the produced mass equal to $\ln \left(M^{2} / m^{2}\right)=Y-Y^{\prime}$. The initial condition for Eq. (2.3) follows from the definition of $N^{D}$ and it has the form with the initial condition given by

$$
N_{i c}^{D}\left(\mathbf{x}_{\perp}, \mathbf{b}, Y_{0}\right)=N^{D}\left(\mathbf{x}_{\perp}, \mathbf{b}, Y=Y_{0}, Y_{0}\right)=0 ; \text { with }-\left.\frac{\partial N^{D}\left(\mathbf{x}_{\perp}, \mathbf{b}, Y, Y_{0}\right)}{\partial Y_{0}}\right|_{Y=Y_{0}}=N_{0}^{2}\left(\mathbf{x}_{\perp}, \mathbf{b}, Y_{0}\right) .
$$

where $N_{0}$ is the solution of the Balitsky - Kovhegov equation [17] and $N_{D}\left(\mathbf{x}, \mathbf{b} ; \mathbf{Y} ; \mathbf{Y}_{\mathbf{0}}\right)$ is the diffraction dissociation of the colourless dipole with size $x_{\perp}$ at impact parameter $b$ into the system of gluons with the rapidity gap larger than $Y_{0}$ at energy $Y$. At first sight, Eq. (2.3) contradicts the AGK relations given by Eq. (2.2) (see Fig. 3), but this contradiction can be easily resolved if we take into account coefficient 2 in Eq. (2.1) (see Refs. [19] for more details as well as for a proof based directly on the dipole approach to high energy scattering).

Despite the simple structure of Eq. (2.3) which is only a little bit more complicated than the Balitsky - Kovhegov equation, as far as we know there exists no analytical solution to this equation and there is the only attempt to solve it numerically 43. However, this equation has a simple solution in the toy model (see Refs. [19, 44, 45] ) which we are going to discuss.

\subsubsection{The BFKL Pomeron calculus in zero transverse dimension.}

The mean field approximation looks extremely simple in the BFKL Pomeron calculus in zero transverse dimension (the toy model [12, 13, 14]). In this model Eq. (2.3) has a simple form

$$
\frac{d N_{D}\left(u ; Y, Y_{0}\right)}{d \Gamma(1 \rightarrow 2) Y}=N_{D}\left(u ; Y, Y_{0}\right)+N_{D}^{2}\left(u ; Y, Y_{0}\right)-4 N_{D}\left(u ; Y, Y_{0}\right) N_{0}(u ; Y)+2 N_{0}^{2}(u ; Y)
$$


Eq. (2.6) gives a typical example of the Liouville equation which has a general solution in the form $N_{0}(u ; Y)=N_{0}(Y+\phi(u))$. Therefore $u$ dependence stems only from the initial conditions of Eq. (2.5) and for $N_{0}(u ; Y)$ we have the Balitsky-Kovchegov equation [17] in a simple form Namely,

$$
\frac{d N_{0}(u ; Y)}{d \Gamma(1 \rightarrow 2) Y}=N_{0}(u ; Y)-N_{0}^{2}(u ; Y)
$$

with the initial condition 12

$$
N_{i c}\left(u, Y_{0}^{\prime}\right)=N_{0}\left(u ; Y=Y_{0}^{\prime}\right)=1-u=\gamma
$$

where $\gamma$ is the amplitude at low energy. Eq. (2.7) has an obvious solution

$$
N_{0}(u ; Y)=\frac{N_{i c}\left(u, Y_{0}^{\prime}\right) \exp (\mathcal{Y})}{1+N_{i c}\left(u, Y_{0}^{\prime}\right)(\exp (\mathcal{Y})-1)}
$$

where $\mathcal{Y}=\Gamma(1 \rightarrow 2) Y$. and $N_{i c}$ are the initial conditions for the scattering amplitude at $Y=Y_{0}^{\prime}$.

To solve Eq. (2.6) we can introduce a new function (see Ref. [19] for details), namely,

$$
N_{i n}\left(u ; Y, Y_{0}\right) \equiv 2 N_{0}(u ; Y)-N_{D}\left(u ; Y, Y_{0}\right)
$$

for which we have the same equation as Eq. (2.7) but with the initial condition given by the following equation:

$$
N_{i c}^{i n}\left(u ; Y_{0}\right) \equiv N_{i n}\left(u ; Y=Y_{0}, Y_{0}\right)=2 N_{i c}\left(u, Y_{0}\right)-N_{i c}^{2}\left(u, Y_{0}\right)
$$

Therefore the solution to Eq. (2.10) with the initial condition of Eq. (2.11) has the form (see Eq. (2.9))

$$
\begin{aligned}
N_{i n}\left(u ; Y, Y_{0}\right) & =\frac{N_{i c}^{i n}\left(u, Y_{0}\right) \exp \left(\mathcal{Y}-\mathcal{Y}_{0}\right)}{1+N_{i c}^{i n}\left(u, Y_{0}\right)\left(\exp \left(\mathcal{Y}-\mathcal{Y}_{0}\right)-1\right)} \\
& =\frac{\left(2 N_{0}\left(u, Y_{0}\right)-N_{0}^{2}\left(u, Y_{0}\right)\right) \exp \left(\mathcal{Y}-\mathcal{Y}_{0}\right)}{1+\left(2 N_{0}\left(u, Y_{0}\right)-N_{0}^{2}\left(u, Y_{0}\right)\right)\left(\exp \left(\mathcal{Y}-\mathcal{Y}_{0}\right)-1\right)}
\end{aligned}
$$

and

$$
N_{D}\left(u ; Y, Y_{0}\right)=2 N_{0}(u ; Y)-N_{i n}\left(u ; Y, Y_{0}\right)
$$

Since $N_{i n}\left(u ; Y, Y_{0}\right)$ satisfies the same equation as $N_{0}\left(u ; Y, Y_{0}\right)$ (see Eq. (2.7)) but with a different initial condition (Eq. (2.11) instead of Eq. (2.8)), one can see that the solution has the following form 19]

$$
N_{\text {in }}\left(u ; Y, Y_{0}\right)=N_{0}(u ; \mathcal{Y}+\Delta \mathcal{Y}) \text { with } \Delta \mathcal{Y}=\ln \left(2+\frac{1-u}{u} \exp \left(\mathcal{Y}_{0}\right)\right)
$$

Using Eq. (2.8) for the initial condition of $N_{0}(u, Y=0)$ and Eq. (2.14) we obtain the following answers for the scattering amplitude, total inelastic contribution and for the diffractive production cross section 
with mass in the interval $\ln \left(M^{2} / m^{2}\right) \leq Y-Y_{0}$ :

$$
\begin{aligned}
& N_{0}(u ; Y) \quad=\frac{(1-u) e^{\mathcal{Y}}}{u+(1-u) e^{\mathcal{Y}}} ; \\
& N_{i n}\left(u ; Y ; Y_{0}\right)=\frac{(1-u) e^{\mathcal{Y}}\left(2 u+(1-u) e^{\mathcal{Y}_{0}}\right)}{u^{2}+(1-u) e^{\mathcal{Y}}\left(2 u+(1-u) e^{\mathcal{Y}_{0}}\right)} ; \\
& N_{S D}\left(u ; Y ; Y_{0}\right)=\frac{u^{2}(1-u)^{2} e^{\mathcal{Y}+\mathcal{Y}_{0}}}{\left(u^{2}+2 u(1-u) e^{\mathcal{Y}}+(1-u)^{2} e^{\left.\mathcal{Y}+\mathcal{Y}_{0}\right)^{2}}\right.} ; \\
& N_{D}\left(u ; Y ; Y_{0}\right)=\frac{2(1-u) e^{\mathcal{Y}}}{u+(1-u) e^{\mathcal{Y}}-\frac{(1-u) e^{\mathcal{Y}}\left(2 u+(1-u) e^{\mathcal{Y}_{0}}\right)}{u^{2}+(1-u) e^{\mathcal{Y}}\left(2 u+(1-u) e^{\mathcal{Y}_{0}}\right)}}
\end{aligned}
$$

in agreement with Refs. 19, 44, 45].

The advantage of our way of obtaining the solution is that it allows us to take into account an arbitrary initial conditions. We will use this in the more general case than the mean field approach.

\subsection{Diffractive production beyond the mean field approximation}

In the kinematic region of rather small produced mass (see Eq. (1.1)) but at very high energies (see Eq. (1.2)) we can neglect the correlations [33, 34, 36] in the diffractive produced system due to the Pomeron loops (see Fig. 2-c, for example).

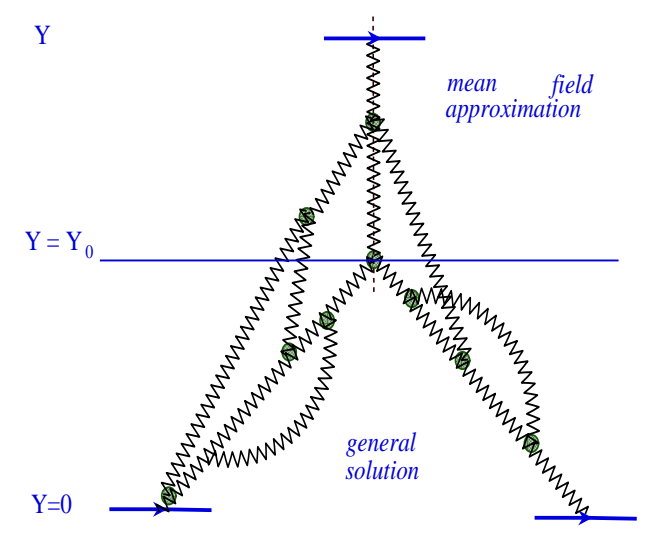

Figure 5: The general diagram for single diffraction production in the kinematic region given by Eq. (1.1) and Eq. (1.2) . The zigzag line crossed by the dotted line denotes the cut Pomeron while the same line without the dotted one stands for the Pomeron exchange.

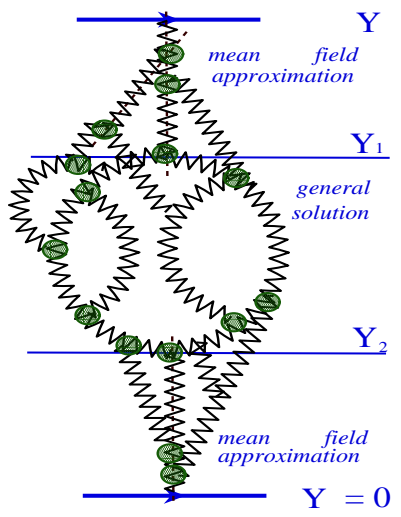

Figure 6: The general diagram for double diffraction production in the kinematic region, given by Eq. (1.1), for both masses $\ln \left(M_{1}^{2} / m^{2}\right)=Y-Y_{1}$ and $\ln \left(M_{2}^{2} / m^{2}\right)=Y_{2}$ and in the energy region, given by Eq. (1.3). The zigzag line crossed by the dotted line denotes the cut Pomeron while the same line without the dotted one stands for the Pomeron exchange. 
The only diagrams that we need to consider are shown in Fig. 5. Our solution, given by Eq. (2.12), sums these diagrams if we substitute in Eq. (2.12) the exact solution of the general equation for $N_{i n}\left(u ; Y_{0}\right)=$ $2 N_{0}\left(u ; Y_{0}\right)$ that includes the Pomeron loops. This equation has the form [15, 28]

$$
\frac{\partial N_{0}\left(u ; \mathcal{Y}_{0}\right)}{\partial \mathcal{Y}_{0}}=u(1-u)\left(-\frac{\partial N_{0}\left(u ; \mathcal{Y}_{0}\right)}{\partial u}+\frac{1}{\kappa} \frac{\partial^{2} N_{0}\left(u ; \mathcal{Y}_{0}\right)}{\partial u^{2}}\right)
$$

where we denote $\mathcal{Y}=\Gamma(1 \rightarrow 2) Y$ and $\kappa=\Gamma(2 \rightarrow 1) / \Gamma(1 \rightarrow 2) . \Gamma(1 \rightarrow 2)$ and $\Gamma(2 \rightarrow 1)$ describe the $P \rightarrow 2 P$ and $2 P \rightarrow P$ transition, respectively.

The initial and boundary conditions are

$$
\begin{aligned}
& \text { Initial conditions: } N_{0}\left(u ; \mathcal{Y}_{0}=0\right)=1-u ; \\
& \text { Boundary conditions: } N_{0}\left(u=0 ; \mathcal{Y}_{0}\right)=1 ; \quad N_{0}\left(u=1 ; \mathcal{Y}_{0}\right)=0 ;
\end{aligned}
$$

Eq. (2.20) stems from the fact that at low energies only the exchange of one Pomeron contributes to the scattering amplitude. The boundary condition at $u=1$ says that the dipole at high energies does not interact with the target if this dipole interacts with zero amplitude at low energies $\left(Y_{0}=0\right)$. At $u=0$ the dipole interacts at low energy with the amplitude which is equal to the maximal value $(N=1)$ and any interaction between Pomerons cannot change this value of the amplitude.
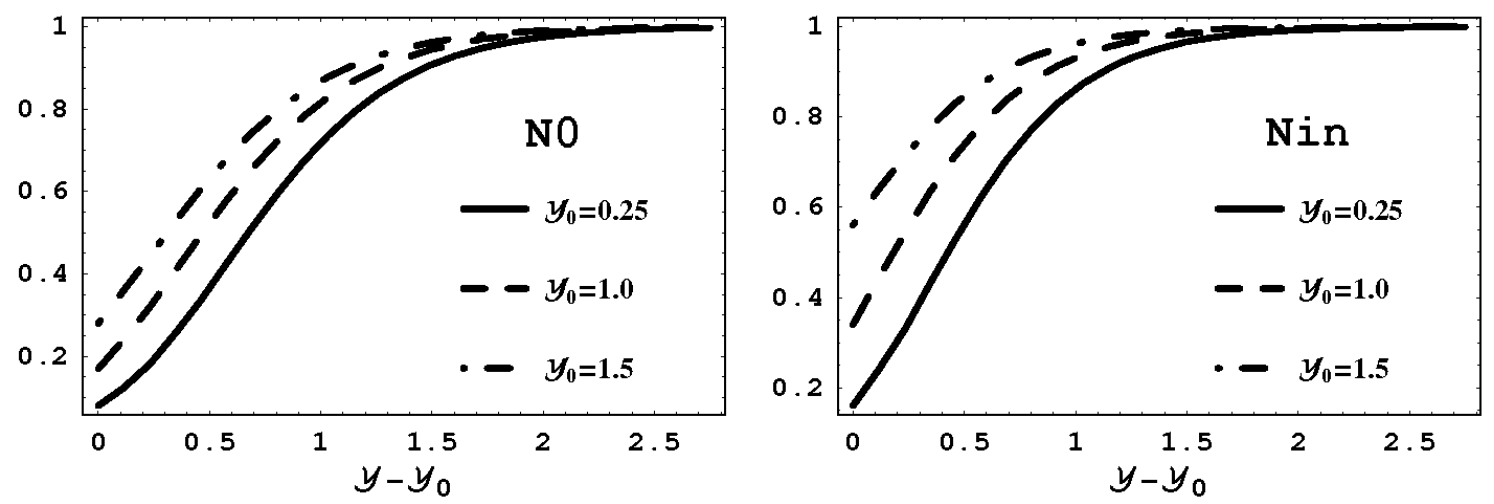

Figure 7: The amplitudes $N_{0}\left(1-u=1 / \kappa, Y ; Y_{0}\right)$ and $N_{i n}\left(1-u=1 / \kappa, Y ; Y_{0}\right)$ as function of $\mathcal{Y}-\mathcal{Y}_{0}=\Gamma(1 \rightarrow$ $2) \cdot\left(Y-Y_{0}\right)$ at different values of $\mathcal{Y}_{0}$

The solution for the single diffractive production can be found within the following procedure:

1. For the cross section of diffractive production of the mass $M(Y=\ln M)$ the initial conditions for the solution of the master equation (see Eq. (2.19)) we put at $Y=Y_{0}$ and they have a form

$$
\begin{aligned}
N_{0}\left(u, Y_{0}\right) & =\frac{(1-u) e^{\mathcal{Y}_{0}}}{1+(1-u)\left(e^{\mathcal{Y}_{0}}-1\right)} ; \\
N_{\text {in }}\left(u, Y_{0}\right) & =\frac{2(1-u) e^{\mathcal{Y}_{0}}}{1+(1-u)\left(e^{\mathcal{Y}_{0}}-1\right)}-\left(\frac{(1-u) e^{\mathcal{Y}_{0}}}{1+(1-u)\left(e^{\mathcal{Y}_{0}}-1\right)}\right)^{2} ;
\end{aligned}
$$



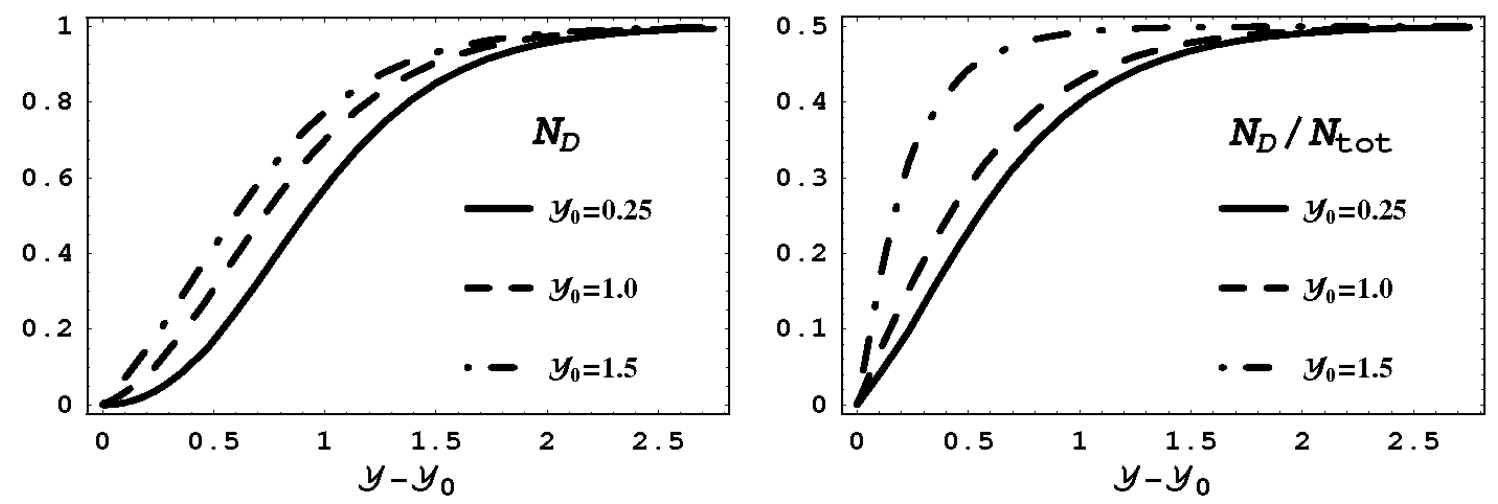

Figure 8: The total cross section of the diffraction dissociation in the range of mass (M) $\ln \left(M^{2} / m^{2}\right) \leq Y_{0}$ $N_{D}\left(1-u=1 / \kappa, Y ; Y_{0}\right)$ and the ratio of this cross section to the elastic amplitude ( the total cross section is equal to $\left.2 N_{0}(1-u=1 / \kappa, Y)\right)$ as function of $\mathcal{Y}-\mathcal{Y}_{0}=\Gamma(1 \rightarrow 2) \cdot\left(Y-Y_{0}\right)$ at different values of $\mathcal{Y}_{0}$
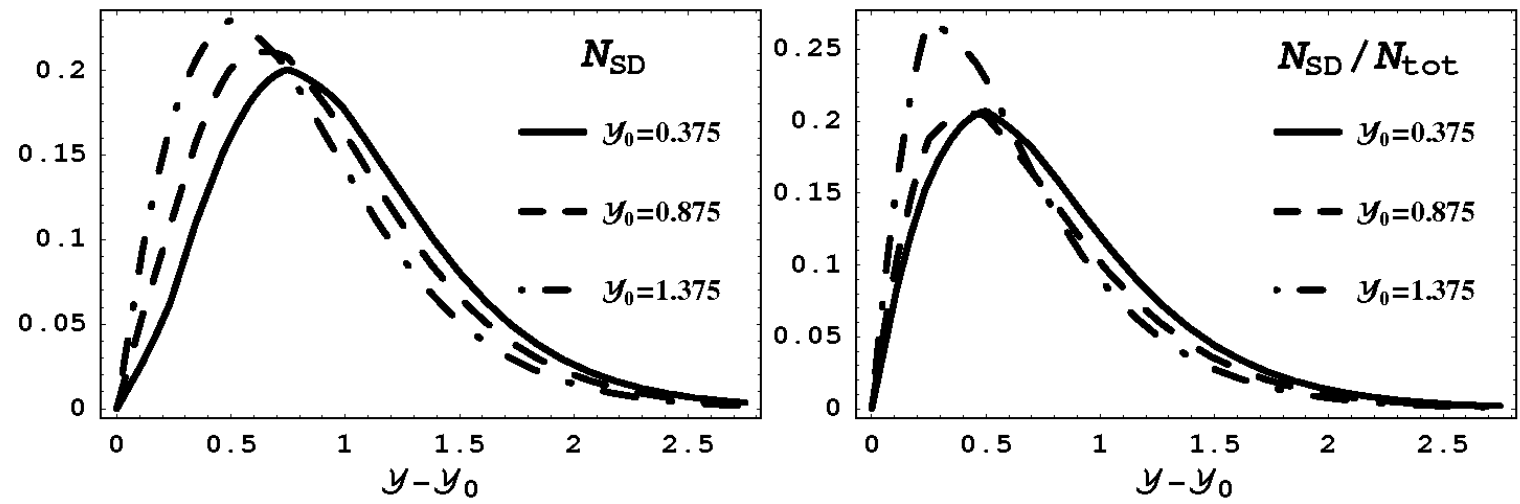

Figure 9: The cross section of the diffraction dissociation at in the range of $\operatorname{mass} \mathcal{Y}_{0, i}+\frac{\Delta \mathcal{Y}_{0}}{2}-\left(\mathcal{Y}_{0, i}-\frac{\Delta \mathcal{Y}_{0}}{2}\right)=\Delta \mathcal{Y}_{0}$ determined by $N_{S D}\left(1-u=1 / \kappa ; Y ; Y_{0, i}\right)=$ $\left(N_{D}\left(1-u=1 / \kappa ; Y ; Y_{0, i}+\frac{\Delta \mathcal{Y}_{0}}{2}\right)-N_{D}\left(1-u=1 / \kappa ; Y ; Y_{0, i}-\frac{\Delta \mathcal{Y}_{0}}{2}\right)\right) / \Delta \mathcal{Y}_{0}$ with $\Delta \mathcal{Y}_{0}=0.25$ and the ratio $N_{S D}\left(1-u=1 / \kappa ; Y ; Y_{0, i}\right) / N_{0}(1-u=1 / \kappa, Y)$ at different values of $\mathcal{Y}_{0, i}$.

2. The next step is to find the exact solution $\left(N_{0}^{\text {exact }}\left(u, Y ; Y_{0}\right)\right.$ and $\left.N_{\text {in }}^{\text {exact }}\left(u, Y ; Y_{0}\right)\right)$ to Eq. (2.19) with the initial conditions determined by Eq. (2.22) and Eq. 2.23). The general method for finding such solution is given in Ref. [28] and the only change that we need to make here is to include different initial condition.

3. However, in Ref. [28] we use Kovchegov's formula 17, 14 for the amplitude, namely,

$$
N_{0}(1-u ; Y)=1-Z(u ; Y)
$$


where $Z$ is the generating function. This formula assumes that $n$-dipoles can interact with one dipole (target) independently. It sums all diagrams of Fig. 2-b type. However ${ }^{1}$, if we would like to restrict ourselves by the enhanced diagrams of Fig. 5 we need to use a different relation between $Z$ and $N$. Namely,

$$
\begin{aligned}
N_{0}\left(1-u=1 / \kappa ; Y ; Y_{0}\right) & =\left.\frac{\partial Z(u ; Y)}{\partial u}\right|_{u=1} \frac{1}{\kappa} ; \\
N_{i n}\left(1-u=1 / \kappa ; Y ; Y_{0}\right) & =\left.\frac{\partial Z_{i n}(u ; Y)}{\partial u}\right|_{u=1} \frac{1}{\kappa} ;
\end{aligned}
$$

In Fig. 7 the solution given by Eq. (2.25) and Eq. (2.26) is plotted as a function of $Y-Y_{0}$ at different values of $Y_{0}$.

4. Using Eq. 2.25) and Eq. (2.26) we can obtain the solution for the single diffraction based on Eq. (2.13), namely,

$$
N_{D}\left(1-u=1 / \kappa ; Y ; Y_{0}\right)=2 N_{0}\left(1-u=1 / \kappa ; Y ; Y_{0}\right)-N_{i n}\left(1-u=1 / \kappa ; Y ; Y_{0}\right)
$$

and for the cross section of single diffraction in the system with mass $Y_{0}=\ln M$ we have

$$
N_{S D}\left(1-u=1 / \kappa ; Y ; Y_{0}\right)=-\frac{d N_{D}\left(1-u=1 / \kappa ; Y ; Y_{0}\right)}{d Y_{0}}
$$

Our calculations for $N_{D}\left(1-u=1 / \kappa ; Y ; Y_{0}\right)$ and $N_{S D}\left(1-u=1 / \kappa ; Y ; Y_{0}\right)$ are shown in Fig. 8 and Fig. 9. The most interesting observation is the fact that in a wide range of produced mass $\mathcal{Y}_{0}<1$ these cross sections only weakly depend on the value of the mass.

\section{Double diffractive production}

In this section we will discuss the double diffractive production, or the reaction of the following type

$$
\begin{aligned}
& \{\text { hadron (dipole) }\}+\{\text { hadron (dipole) }\} \Longrightarrow \\
& \left\{\text { hadron(gluon) system with mass } M_{1}\right\}+\{\text { LRG }\}+\left\{\text { hadron(gluon) system with mass } M_{2}\right\}
\end{aligned}
$$

where both masses $\left(M_{1}\right.$ and $\left.M_{2}\right)$ satisfy the relation of Eq. (1.2) while the total energy is so large that Eq. (1.3) holds. The diagrams that contribute to this process in the kinematic region, that we have mentioned, are shown in Fig. 6. For the double diffraction production in the kinematic region, given by Eq. (1.1), for both masses $\ln \left(M_{1}^{2} / m^{2}\right)=Y-Y_{1}$ and $\ln \left(M_{2}^{2} / m^{2}\right)=Y_{2}-0$ and in the energy region, given by Eq. (1.3), we can neglect the Pomeron loops contributions in the region $Y-Y_{1}$ and $Y_{2}-0$ (see Fig. 6). This simplification leads to a simple procedure of calculating the cross section for the double diffractive dissociation, which consists of four steps:

\footnotetext{
${ }^{1}$ We thank Edmond Iancu and Al Mueller for hot discussions on this subject.
} 
1. As the initial conditions for the double diffractive processes we choose Eq. (2.22) and Eq. (2.23) at $Y_{0}=Y_{2}$. They give us $N_{i n}\left(u, Y_{2}\right)$ and $N_{0}\left(u, Y_{2}\right)$;

2. Using the general method of Ref. [28], we find the generating function $Z\left(u, Y_{1}-Y_{2} ; Y_{2}\right)$ and $Z_{\text {in }}\left(u, Y_{1}-Y_{2} ; Y_{2}\right)$ as the solution of Eq. (2.19) with the initial conditions

$$
\begin{aligned}
Z_{i n}\left(u, Y_{1}-Y_{2}=0 ; Y_{2}\right) & =1-N_{i n}\left(u, Y_{2}\right) ; \\
Z\left(u, Y_{1}-Y_{2}=0 ; Y_{2}\right) & =1-N_{0}\left(u, Y_{2}\right) ;
\end{aligned}
$$

3. The third step is to notice that the amplitudes $N_{0}\left(u, Y-Y_{1}\right)$ and $N_{i n}\left(u, Y-Y_{1}\right)$ are also determined by Eq. (2.22) and Eq. (2.23) at $Y_{0}=Y-Y_{1}$

4. The fourth and the last step is to find the final answer using the general formula (see Ref. [33, 34, 13, 14 ) that was re-written in the compact form in Ref. 35]

$$
\begin{aligned}
& N_{0}\left(\gamma=1 / \kappa, Y-Y_{1} ; Y_{1} ; Y_{2}\right)= \\
& 1-\left.\left\{\exp \left(-\gamma \frac{d}{d u_{1}} \frac{d}{d u_{2}}\right) N_{0}\left(Y-Y_{1}, u_{1}\right) Z\left(Y_{1} ; Y_{2}, u_{2}\right)\right\}\right|_{u_{1}=1 ; u_{2}=1} ; \\
& N_{i n}\left(\gamma=1 / \kappa, Y-Y_{1} ; Y_{1} ; Y_{2}\right)= \\
& 1-\left.\left\{\exp \left(-\gamma \frac{d}{d u_{1}} \frac{d}{d u_{2}}\right) N_{i n}\left(Y-Y_{1}, u_{1}\right) Z_{i n}\left(Y_{1} ; Y_{2}, u_{2}\right)\right\}\right|_{u_{1}=1 ; u_{2}=1} ;
\end{aligned}
$$

We can simplify Eq. (3.4) and Eq. (3.5) going to the Mellin transform for the generating functions $Z_{\text {in }}\left(Y_{1} ; Y_{2}, u_{2}\right)$ and $Z\left(Y_{1} ; Y_{2}, u_{2}\right)$. Namely,

$$
Z\left(Y_{1} ; Y_{2}, u\right)=\int_{a-i \infty}^{a+i \infty} \frac{d \mu}{2 \pi i} e^{\mu \ln (1 / u)} \tilde{Z}\left(Y_{1} ; Y_{2}, \mu\right)
$$

Since $d^{n} N_{0}\left(Y-Y_{1}, u_{1}\right) / d^{2} u_{1}$ is known, namely,

$$
\frac{d^{n} N_{0}\left(Y-Y_{1}, u_{1}\right)}{d u_{1}^{n}}=n ! e^{\mathcal{Y}-\mathcal{Y}_{1}} \times\left(e^{\mathcal{Y}-\mathcal{Y}_{1}}-1\right)^{n-1}
$$

Eq. (3.4) reads as

$$
\begin{aligned}
& N_{0}\left(\gamma, Y-Y_{1} ; Y_{1} ; Y_{2}\right)= \\
& \int_{a-i \infty}^{a+i \infty} \frac{d \mu}{2 \pi i} \tilde{Z}\left(Y_{1} ; Y_{2}, \mu\right) \frac{e^{\mathcal{Y}-\mathcal{Y}_{1}}}{\left(e^{\mathcal{Y}-\mathcal{Y}_{1}}-1\right)}\left\{-\Gamma\left(1-\mu, \frac{1}{\gamma T}\right)\left(\frac{1}{\gamma T}\right)^{\mu} \exp \left(\frac{1}{\gamma T}\right)+1\right\}
\end{aligned}
$$

where $T \equiv\left(e^{\mathcal{Y}-\mathcal{Y}_{1}}-1\right)$ and $\gamma=1 / \kappa$. Since (see Ref. 49 formula 3.381(3))

$$
\int_{1}^{\infty} d x x^{-\mu} \exp \left(-\frac{x}{\gamma T}\right)=\left(\frac{1}{\gamma T}\right)^{\mu-1} \Gamma\left(1-\mu, \frac{1}{\gamma T}\right)
$$


one can see that Eq. (3.8) can be re-written in the form

$$
\begin{aligned}
& N_{0}\left(\gamma, Y-Y_{1} ; Y_{1} ; Y_{2}\right) \times\left(\frac{e^{\mathcal{Y}-\mathcal{Y}_{1}}-1}{e^{\mathcal{Y}-\mathcal{Y}_{1}}}\right)= \\
& -\gamma T \exp \left(\frac{1}{\gamma T}\right) \int_{1}^{\infty} d x \exp \left(-\frac{x}{\gamma T}\right) \int_{a-i \infty}^{a+i \infty} \frac{d \mu}{2 \pi i} x^{-\mu} \tilde{Z}\left(Y_{1} ; Y_{2}, \mu\right)+1= \\
& -\gamma T \exp \left(\frac{1}{\gamma T}\right) \int_{1}^{\infty} x d x \exp \left(-\frac{x}{\gamma T}\right) Z\left(Y_{1} ; Y_{2}, \ln (1 / x)\right)+1= \\
& -\gamma T \exp \left(\frac{1}{\gamma T}\right) \int_{0}^{1} \frac{d u}{u^{2}} \exp \left(-\frac{1}{u \gamma T}\right) Z\left(Y_{1} ; Y_{2}, u\right)+1
\end{aligned}
$$

Eq. (3.10) is convenient for calculation. In derivation of this equation we used that $Z\left(Y_{1} ; Y_{2}, u=1\right)=1$ (see Eq. (2.21)).

For $N_{\text {in }}\left(\gamma, Y-Y_{1} ; Y_{1} ; Y_{2}\right)$ we obtain a reasonable approximation by using the same expression as Eq. (3.10) but with $T \rightarrow 2 T$ and $Z \rightarrow Z_{\text {in }}$. Indeed, in Eq. (2.23) we can consider $1-u \approx 1 / \kappa \ll 1$ and neglect the second term in Eq. (2.23) if $(1-u) \exp \left(\mathcal{Y}-\mathcal{Y}_{0}\right) \ll 1$ or $\mathcal{Y}-\mathcal{Y}_{0} \ll 1 /(1-u) \approx \kappa$. Recall that this interval for $\mathcal{Y}-\mathcal{Y}_{0}$ is much wider than the one given by Eq. (1.1), since $\kappa=1 / \alpha_{S}^{2}$.

Finally, the inclusive double diffractive process, namely, the cross section defined as

$$
N_{i n}^{D D}=\int_{0}^{Y-Y_{1}} d \ln \left(M_{1}^{2} / m^{2}\right) \int_{0}^{Y_{2}} d \ln \left(M_{2}^{2} / m^{2}\right) M_{1}^{2} M_{2}^{2} \frac{d \sigma}{d M_{1}^{2} d M_{2}^{2}}
$$

we calculate as

$$
N_{i n}^{D D}\left(u ; Y, Y_{1}, Y_{2}\right)=2 N_{0}(u, Y)-N_{i n}\left(u ; Y, Y_{1}, Y_{2}\right)
$$

For calculation of the double diffraction cross section in the two system of hadrons with fixed mass we need

$$
M_{1}^{2} M_{2}^{2} \frac{d \sigma}{d M_{1}^{2} d M_{2}^{2}}=-\frac{d N_{i n}^{D D}\left(u ; Y, Y_{1}, Y_{2}\right)}{d Y_{1} d Y_{2}}
$$

\section{Survival probability for the central diffractive production.}

In our approach the value, that we call survival probability, is defined as the following ratio:

$$
<\left|S^{2}\right|>=\frac{\mid\left. A(\text { Fig. } 10-a)\right|^{2}}{\mid\left. A(\text { Fig. } 10-b)\right|^{2}}
$$

This ratio is the quantitative measure how the interactions of the 'wee' partons from different parton showers are suppressed the probability of the production of the central system, which is produced with rapidities close to $Y / 2$ in the reaction of Eq. (1.4) type.

To find the amplitude for the diagram of Fig. 10-a we need to take into account that the Higgs meson in Fig. 10 can be emitted from any of Pomerons at rapidity $Y / 2$ in this diagram. We can take into account 


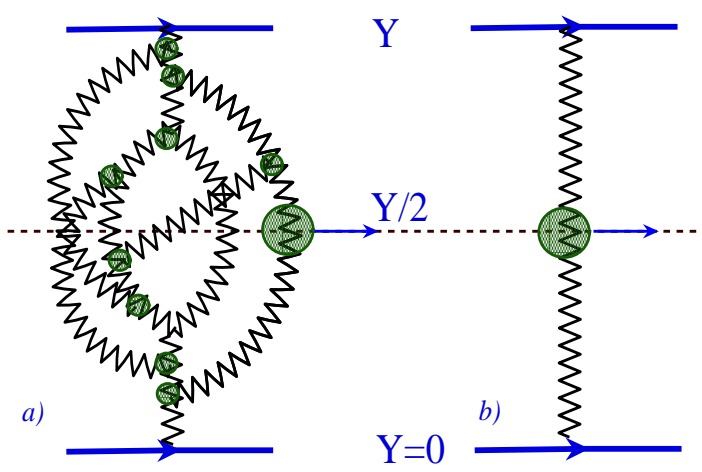

Figure 10: The central diffractive Higgs meson production: diagrams Fig. 10-a is a general case that takes into account the interaction of many parton showers, while the diagram of Fig. 10-b is the central production from one parton shower.

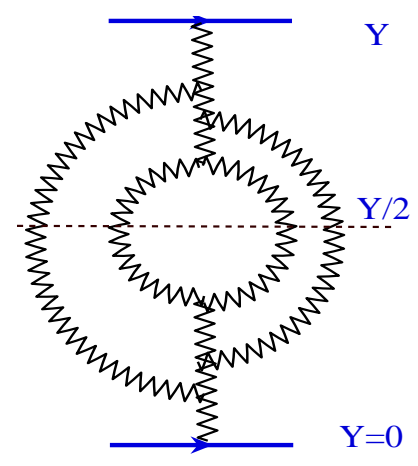

Figure 11: The diagrams that contribute in Mueller-Patel-Salam-Iancu approximation, which describe the amplitude for the rapidities that satisfy the restriction $Y \equiv \ln \left(M^{2} / m^{2}\right) \ll \frac{1}{\bar{\alpha}_{S}} \ln \left(\frac{N_{c}^{2}}{\bar{\alpha}_{S}^{2}}\right)$.

this fact by the following procedure. First, we recall that the scattering amplitude can be written in the form [13, 14, 17]

$$
N\left(\gamma ; \frac{Y}{2}\right)=-\left.\sum_{n=1}^{\infty} \frac{(-1)^{n}}{n !} \gamma^{n} \frac{\partial^{n} Z(u, Y)}{\partial^{n} u}\right|_{u=1}=-\sum_{n=1}^{\infty}(-1)^{n} \gamma^{n} \rho_{n}
$$

Therefore, to calculate the Higgs production we need to take the following derivative

$$
\gamma \frac{\partial N(\gamma ; Y)}{\partial \gamma}=-\sum_{n=1}^{\infty} n(-1)^{n} \gamma^{n} \rho_{n}
$$

The next step is to solve our master equation (see Eq. (2.19)) using Eq. (4.3) as the initial condition. This solution $\left(N\left(\gamma ; Y ; \frac{Y}{2}\right)\right)$ will allow us to find the survival probability. For calculating the survival probability we need to use the equation

$$
<\left|S^{2}\right|>=\left(\frac{N\left(\gamma ; Y ; \frac{Y}{2}\right)}{\gamma \exp (\mathcal{Y})}\right)^{2}
$$

In Fig. 12 our estimates for the value of the survival probability is plotted versus energy. We need to know the value of the $P \rightarrow 2 P$ vertex to assign the measured value of the total rapidity $Y=\ln \left(s / s_{0}\right)$ with $s_{0} \approx 1 \mathrm{GeV}^{2}$. Since this value is very important characteristic of the diffractive Higgs production we present the result of calculation in Table 1 . If we take $\Gamma(1 \rightarrow 2)=\Delta_{P}(0)$ where $\Delta_{P}(0)$ is the intercept of soft Pomeron $\left(\Delta_{P}(0) \approx 0.1\right)$ the value of $\left\langle\left|S^{2}\right|\right\rangle \approx 0.23$. This value is in agreement with the estimates based on the two channel model for diffractive production [37, 39]. However, if $\Delta_{P}(0) \approx \bar{\alpha}_{S}=0.25$ the value of $\left\langle\left|S^{2}\right|\right\rangle=7 \cdot 10^{-4}$ which is much smaller. It should be stressed that our calculations are one of the first that includes the enhanced diagrams (Pomeron loops) and can be used to evaluated the effect of this contribution on the value of the survival probability. One can see that the effect can be very large and the 


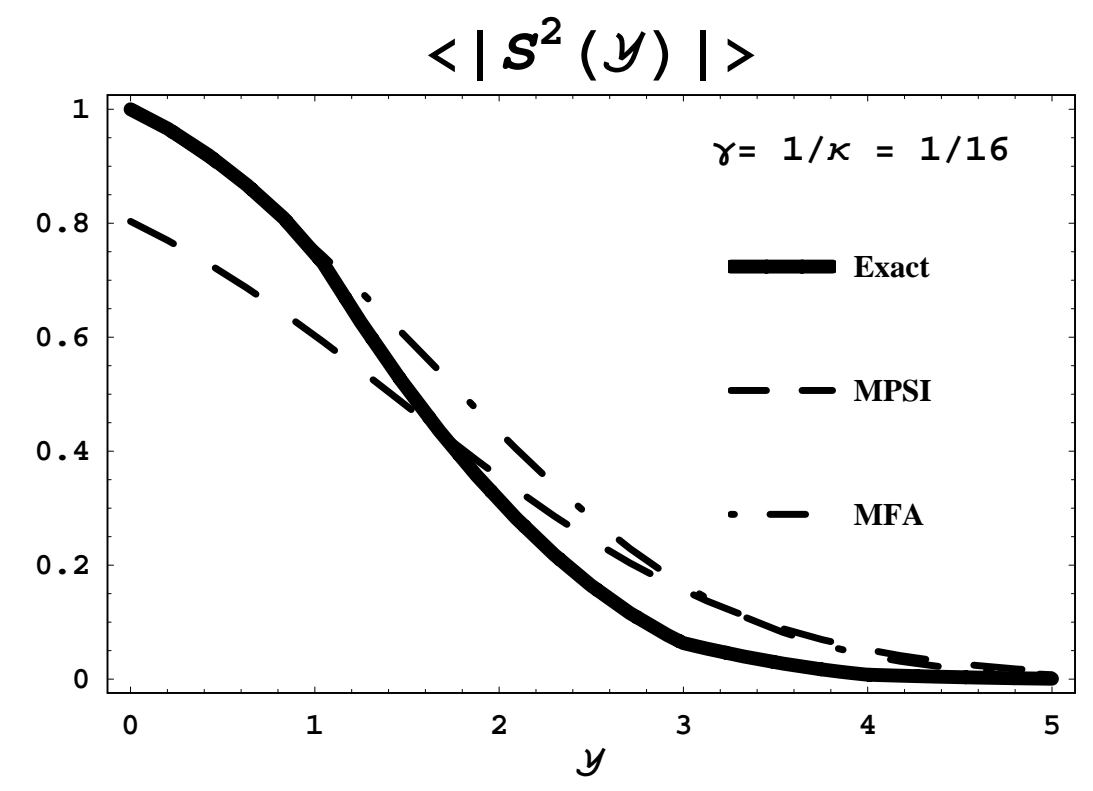

Figure 12: Survival probability $\left\langle\mid S^{2}\right\rangle$ versus $\mathcal{Y} \equiv \Gamma(1 \rightarrow 2) Y$ at different values for the low energy amplitude $\gamma$. The solid line is our exact calculation (Eq. (4.4)) while the dotted line is the MPSI approximation (Eq. (5.13)) and the dashed-dotted line shows the calculation in the MFA (Eq. (5.3))

\begin{tabular}{|l|l|l|l|l|l|l|l|l|l|l|}
\hline \hline $\mathcal{Y}$ & 0 & 0.25 & 0.5 & 0.75 & 1. & 1.25 & 1.5 & 1.75 & 2. & 2.25 \\
\hline$\left\langle\left|S^{2}\right|\right\rangle$ & 1 & 0.96 & 0.83 & 0.75 & 0.62 & 0.751 & 0.41 & 0.31 & 0.23 & 0.16 \\
\hline \hline $\mathcal{Y}$ & 2.5 & 2.75 & 3. & 3.25 & 3.75 & 4. & 4.25 & 4.5 & 4.75. & 5. \\
\hline$\left\langle\left|S^{2}\right|\right\rangle$ & 0.11 & 0.063 & 0.045 & 0.029 & 0.017 & 0.0077 & 0.0054 & 0.0034 & 0.0018 & 0.0007 \\
\hline \hline
\end{tabular}

Table 1: The values of the survival probabilities versus $\mathcal{Y}=\Gamma(1 \rightarrow 2) Y$ at the value of the low energy amplitude $\gamma=1 / \kappa$.

process of the diffractive Higgs production could be much less than it is expected based on the estimates in Refs. [37, 38, 39].

The estimates, discussed above, have one obvious defficientcy: they do not take into account the fact that the Higgs meson production cannot occur for energies less than $s=M^{2}$ where $M$ is the mass if Higgs meson. In other language, the vertex for Higgs meson production has a size in rapidity while we neglected this effect in Eq. (4.4). It is easy to take into account this replacing Eq. (4.4) by

$$
<\left|S^{2}\right|>=\left(\frac{N\left(\gamma ; Y ; \frac{Y}{2}\right)}{N\left(\gamma ; \zeta ; \frac{\zeta}{2}\right)}\right)^{2} \text { with } \zeta=\ln \left(\frac{M_{\text {Higgs }}^{2}}{s_{0}}\right)
$$




\begin{tabular}{|l|l|l|l|l|l|l|l|l|l|l|}
\hline \hline $\mathcal{Y}$ & 2.5 & 2.75 & 3. & 3.25 & 3.75 & 4. & 4.25 & 4.5 & 4.75. & 5. \\
\hline$\left\langle\left|S^{2}\right|\right\rangle$ & 0.69 & 0.39 & 0.28 & 0.18 & 0.10 & 0.048 & 0.034 & 0.021 & 0.010 & 0.0044 \\
\hline \hline
\end{tabular}

Table 2: The values of the survival probabilities versus $\mathcal{Y}=\Gamma(1 \rightarrow 2) Y$ (the value of the low energy amplitude $\gamma=1 / \kappa$.) Calculating $\left\langle\left|S^{2}\right|\right\rangle$ Eq. (4.5) was used with $\alpha_{S}=1 / 4$ and $M_{\text {Higgs }}=100 \mathrm{GeV}$.

In the Table 2 we show values of $\left\langle\left|S^{2}\right|>\right.$ for $\bar{\alpha}_{S}=0.25$, when calculations ware archived using Eq. (4.5). One can see that the difference between Eq. (4.4) and Eq. (4.5) (Table 1 and Table 2 correspondingly) is large and expected $\left\langle\left|S^{2}\right|\right\rangle$ at the LHC energy is equal to $4.4 \cdot 10^{-3}$ instead of $\left\langle\left|S^{2}\right|\right\rangle=7 \cdot 10^{-4}$ The conclusion that we want to make is that the enhanced Pomeron diagrams can be very important in obtaining the reliable estimates of the survival probability of the large rapidity gap at the LHC energy and can lead to additional suppression of the value of the cross section for diffractive Higgs production.

\section{Comparison with the approximate approaches.}

\subsection{Our approach versus the mean field approximation.}

In the mean field approximation we can discuss the single diffractive production (see Eq. (2.15) - Eq. (2.18)) and the value of the survival probability. The direct application of the procedure described in Eq. (4.2) Eq. (4.4) for the MFA gives

$$
\begin{aligned}
& \gamma \frac{\partial N(\gamma ; Y)}{\partial \gamma}=\frac{\exp \left(\frac{\mathcal{Y}}{2}\right)}{\left(1+\gamma\left(\exp \left(\frac{\mathcal{Y}}{2}\right)-1\right)\right)^{2}} ; \\
& N\left(\gamma ; Y ; \frac{Y}{2}\right)=\frac{\gamma \exp (\mathcal{Y})}{\left(1+\gamma\left(\exp \left(\frac{\mathcal{Y}}{2}\right)-1\right)\right)^{2}+\gamma \exp \left(\frac{\mathcal{Y}}{2}\right)\left(\exp \left(\frac{\mathcal{Y}}{2}\right)-1\right)} \\
& <\left|S^{2}\right|>=\left(\frac{1}{\left(1+\gamma\left(\exp \left(\frac{\mathcal{Y}}{2}\right)-1\right)\right)^{2}+\gamma \exp \left(\frac{\mathcal{Y}}{2}\right)\left(\exp \left(\frac{\mathcal{Y}}{2}\right)-1\right)}\right)^{2} ;
\end{aligned}
$$

However, to compare the MFA with our calculations we need to go back from Eq. (2.25) and Eq. (2.26) to Kovchegov's formulae

$$
\begin{aligned}
& N_{0}(\gamma ; Y)=1-Z(1-\gamma ; Y) \\
& N_{i n}(\gamma ; Y)=1-Z_{i n}(1-\gamma ; Y)
\end{aligned}
$$

The comparison with the MFA is given in Fig. 14 and Fig. 13 for the diffractive cross section and survival probability. As it is expected the MFA reproduces the exact result only for rather large values of the amplitude for dipole - target interaction at low energies. 

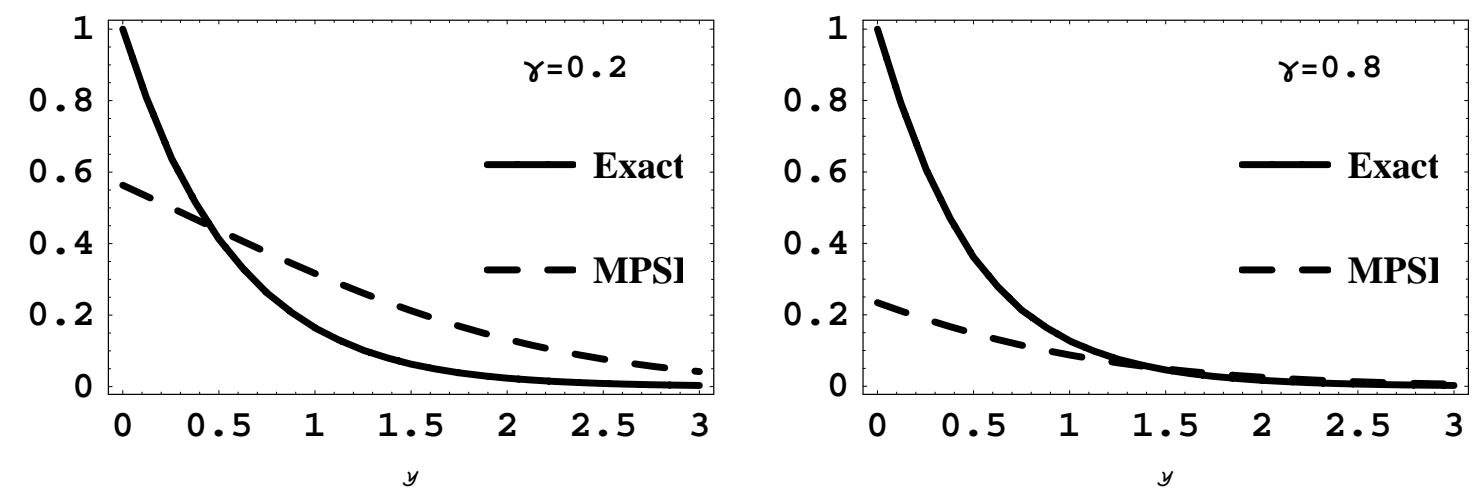

Figure 13: Survival probability $\left\langle\mid S^{2}\right\rangle$ versus $\mathcal{Y} \equiv \Gamma(1 \rightarrow 2) Y$ at different values for the low energy amplitude $\gamma$. The solid line is our calculation while the dotted line is the MFA approximation.

\subsection{Our approach versus Mueller - Patel - Salam - Iancu (MPSI) approximation.}

The MPSI approximation [31, 32, 33, 34] replaces the mean field one in the case if the dipole - target amplitude is small at low energies. The diagrams that contribute to the scattering amplitude in this approximation are shown in Fig. 11, they all have Pomeron loops but the size of any loop is larger than $Y / 2$. The general formula of MPSI approximation can be written in the form

$$
\begin{aligned}
N_{0}^{M P S I}(\gamma, Y) & =1-\left\{\exp \left(-\gamma \frac{d}{d u_{1}} \frac{d}{d u_{2}}\right) N_{0}^{M F A}\left(\frac{Y}{2}, u_{1}\right) N_{0}^{M F A}\left(\frac{Y}{2}, u_{2}\right)\right\} \mid u_{1}=1 ; u_{2}=1 \\
& =1-\exp \left(\frac{1}{\gamma e^{\mathcal{Y}}}\right) \frac{1}{\gamma e^{\mathcal{Y}}} \Gamma\left(0, \frac{1}{\gamma e^{\mathcal{Y}}}\right)
\end{aligned}
$$

$\Gamma(0, x)$ is the incomplete gamma function (see formulae 8.350 - $\mathbf{8 . 3 5 9}$ in Ref. 490). We assume that $e^{\mathcal{Y} / 2} \gg 1$ in Eq. (5.6). In Eq. (5.6) we substitute for $N_{0}^{M F A}$ the amplitude in the mean field approximation but we can use this equation using any of the amplitude calculated in Eq. (2.15) - Eq. (2.18). For example if one needs to calculate the single diffraction production Eq. (5.6) has the form

$$
\begin{aligned}
N_{S D}^{M P S I}\left(\gamma, Y, Y_{0}\right) & =1-\left.\left\{\exp \left(-\gamma \frac{d}{d u_{1}} \frac{d}{d u_{2}}\right) N_{S D}^{M F A}\left(\frac{Y}{2}, u_{1}\right) \tilde{N}_{0}^{M F A}\left(\frac{Y}{2}, u_{2}\right)\right\}\right|_{u_{1}=1 ; u_{2}=1} \\
& =\frac{2 e^{\mathcal{Y}-\mathcal{Y}_{0}}}{T_{1}^{2}}\left\{\frac{1}{\gamma T_{1}}+1-\frac{1+2 \gamma T_{1}}{\left(\gamma T_{1}\right)^{2}} \Gamma\left(0, \frac{1}{\gamma T_{1}}\right) \exp \left(\frac{1}{\gamma T_{1}}\right)\right\}
\end{aligned}
$$

where $T_{1} \equiv T\left(Y, Y_{0}\right)=2 \exp (\mathcal{Y})-\exp \left(\mathcal{Y} / 2+\mathcal{Y}_{0}\right)$

In derivation of Eq. (5.7) we use not Eq. (2.17) but simpler expression, namely [44, 45]

$$
\tilde{N}_{0}^{M F A}\left(Y, Y_{0}\right)=\frac{2(1-u) e^{\mathcal{Y}}\left(2 e^{\mathcal{Y}}-e^{\mathcal{Y}_{0}}-1\right)}{\left(1+(1-u)\left(2 e^{\mathcal{Y}}-e^{\mathcal{Y}_{0}}-1\right)\right)^{2}}
$$



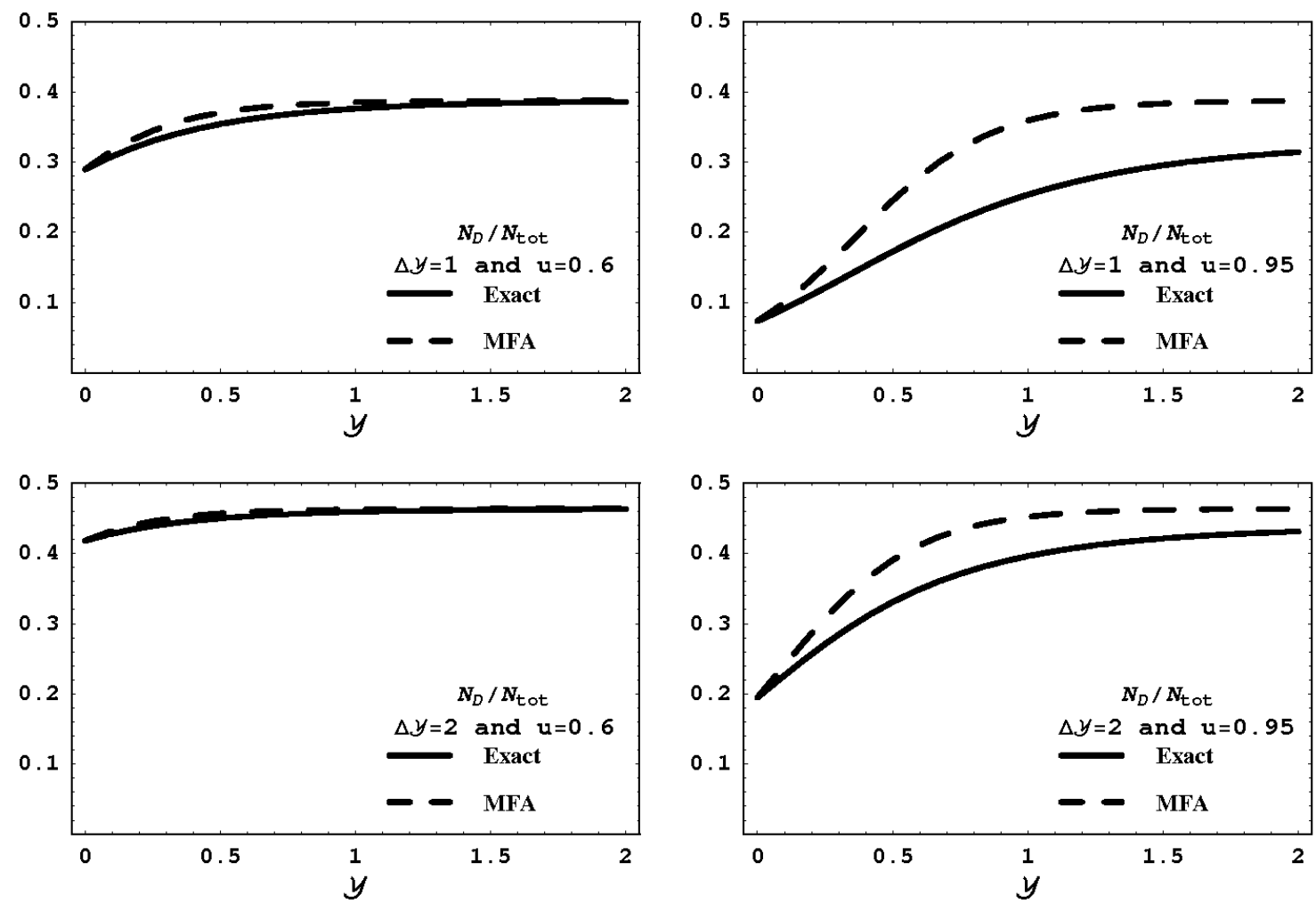

Figure 14: The ratio $N_{D} / N_{0}$ as a function of $\mathcal{Y}$ for different value of the amplitude of interaction for low energies $\gamma=1-u$. The solid line is our calculation while the dotted line is the MFA approximation.

This simple formula does not take into account the elastic scattering of the dipole while Eq. (2.17) does. Therefore, we find in Eq. (5.7) the equation for the inelastic diffraction. Practically, such approach could be useful for the diffraction in hadron-hadron interaction, however, it is certainly is not good approximation for deep inelastic scattering where the elastic scattering also included in the experimental diffractive cross se3ction.

Substituting in Eq. (5.7) $\tilde{N}_{D}^{M F A}$ given by

$$
\tilde{N}_{D}^{M F A}=2(1-u) e^{\mathcal{Y}}\left(\frac{1}{1++(1-u)\left(e^{\mathcal{Y}}-\right)}-\frac{1}{1+(1-u)\left(2 e^{\mathcal{Y}}-e^{\mathcal{Y}_{0}}-1\right)}\right)
$$

we obtain

$$
\begin{aligned}
& N_{D}^{M P S I}\left(\gamma ; Y, Y_{0}\right)= \\
& 2\left\{1-\frac{1}{\gamma e^{\mathcal{Y}}} \exp \left(\frac{1}{\gamma e^{\mathcal{Y}}}\right) \Gamma\left(0, \frac{1}{\gamma e^{\mathcal{Y}}}\right)-\frac{e^{\mathcal{Y}}}{T_{1}}\left(1-\exp \left(\frac{1}{\gamma T_{1}}\right) \frac{1}{\gamma T_{1}} \Gamma\left(0, \frac{1}{\gamma T_{1}}\right)\right)\right\}
\end{aligned}
$$


Repeating the same calculation but for double diffraction using Eq. (5.7) but with substitution $N_{0} \rightarrow$ $N_{S D}$ we obtain the following

$$
\begin{aligned}
& N_{D D}^{M P S I}\left(\gamma, Y, Y_{1}, Y_{2}\right)= \\
& 4 \frac{e^{\mathcal{Y}-\mathcal{Y}_{1}-\mathcal{Y}_{2}}}{T_{2}^{2}} \times\left\{\frac{1}{\left(\gamma T_{2}\right)^{2}}+\frac{4}{\gamma T_{2}}+1-\frac{1}{\gamma T_{2}} \Gamma\left(0, \frac{1}{T_{2}}\right) \times \exp \left(\frac{1}{\gamma T_{2}}\right)\left[\frac{1}{\left(\gamma T_{2}\right)^{2}}+\frac{5}{\gamma T_{2}}+4\right]\right\}
\end{aligned}
$$

where $T_{2} \equiv T\left(Y, Y_{1}, Y_{2}\right)=\left(2 \exp \left(\frac{\mathcal{Y}}{2}\right)-\exp \left(\mathcal{Y}_{1}\right)\right)\left(2 \exp \left(\frac{\mathcal{Y}}{2}\right)-\exp \left(\mathcal{Y}_{2}\right)\right)$.

Finally, for the survival probability we obtain in MPSI approach the following expression

$$
<\left|S^{2}\right|>=-\frac{1}{\left(\gamma e^{\mathcal{Y}}\right)^{2}}+\frac{1+\gamma e^{\mathcal{Y}}}{\left(\gamma e^{\mathcal{Y}}\right)^{3}} \exp \left(\frac{1}{\gamma e^{\mathcal{Y}}}\right) \Gamma\left(0, \frac{1}{\gamma e^{\mathcal{Y}}}\right)
$$

In writing Eq. (5.13) we assume that $e^{\mathcal{Y}} \gg 1$.

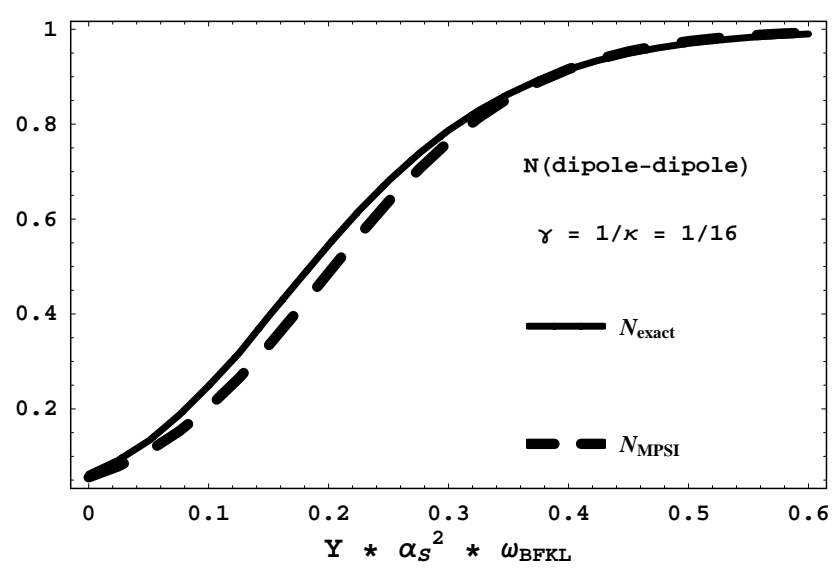

Figure 15: The comparison of the scattering amplitude for the dipoledipole scattering $\left(N_{0}\right)$ calculated using Eq. (2.25). The $x$-axis is $\mathcal{Y}=$ $\omega_{B F K L} \alpha_{S}^{2} Y=\left(4 \ln 2 \bar{\alpha}_{S} / \kappa\right) Y$. The solid line is the exact solution while the dotted one shows the MPSI result.

our amplitude. In Fig. 15 one can see the result of these calculation. One can see from this figure that the MPSI approach describes quite well the high energy limit of the scattering amplitude but it underestimates the value of the amplitude at lower energies. This result is actually quite surprising since the MPSI approach was proven to be a good approximation in the limited range of energies, namely,

$$
\frac{1}{\bar{\alpha}_{S}} \ll Y \ll \frac{1}{\bar{\alpha}_{S}} \ln \left(\frac{N_{c}^{2}}{\bar{\alpha}_{S}^{2}}\right)
$$


For our choice of $\kappa=16$ and $\alpha_{S}=1 / 4$ in Fig. 15 ( considering $\omega_{B F K L}=4 \ln 2 \bar{\alpha}_{S}$ ) Eq. (5.14) gives $0.044<\mathcal{Y}=\left(\omega_{B F K L} / \kappa\right) Y<0.2$. One can see that in this range the MPSI approximation is not able to describe the exact solution.

In Fig. 16 we see that even for the single diffractive production the MPSI approximation describes the exact result quite well.

\section{Conclusions}

In this paper we obtain two main results. The first result of this paper is the set of formulae in the framework of the BFKL Pomeron calculus in zero transverse dimensions, that describe the diffractive dissociation processes in the limited range of produced mass given by Eq. (1.1) - Eq. (1.3). The comparison with the approximation methods, that there exists on the market, shows that these methods are not able to describe the diffractive production in the entire kinematic region. However, the mean field approximation gives a very good description if the low energy amplitude of interaction with the target is rather large and if the two or more dipoles interact with the target without correlations according to the Glauber formulae.

This great surprise stems from the fact that the MSPI approximation is able to describe the high energy limit of the amplitudes for elastic and diffractive scattering while theoretically this approach can be quaranteed only in the limited kinematic range given by Eq. (5.14).

The surprise comes from two observations: first, the MSPI result does not lead to the decreasing cross section in spite of the fact that onle triple Pomeron interaction has been taken into account; and, second, for the total cross section the MSPI estimates are very close to the exact solution (see Fig. 15).

Having in mind these two observations we believe that the MSPI approximation can be used as the simple first approximation instead of the MFA for the case of dipoloe-dipole scattering. However4, we would like to stress that for the value of the survival probability the MPSI approximation gives leads to higher probability of survival of the large rapidity gaps than the exact answer.

The second result is the formula for the survival probability for the central diffractive (see Eq. (1.4) production of Higgs meson (di-jets and so on ). This formula is exact and it is valid for any value of energy. The estimates using this formula gives the value for the survival probability which is much smaller (in 10 - 100 times) than the estimates of all approximation methods that have been used before. We hope that this result will stimulate a more detailed study of the survival probability in QCD and we consider the first papers on this subject (see Refs. 40, 42] as only a beginning for such kinds of studies.

In general, we hope that this paper, devoted to a model: the BFKL Pomeron calculus in zero transverse dimension, will be useful in searching the high energy asymptotic behaviour in the general case of QCD and/or in the case of the BFKL Pomeron calculus in two transverse dimensions.

We would like to draw attention to the fact that in this paqper we sum mostly the so called emhanced diagrams (see Eq. (2.25) and Eq. (2.26) and Fig. 5,Fig. 6 and Fig. 10). These diagrams cannot give the correct answer since they did not reproduce the elastic cross section. The elastic cross section is equal to zero in this approach. However, accordingly to the general idea of the Pomeron calculus the sum of these 

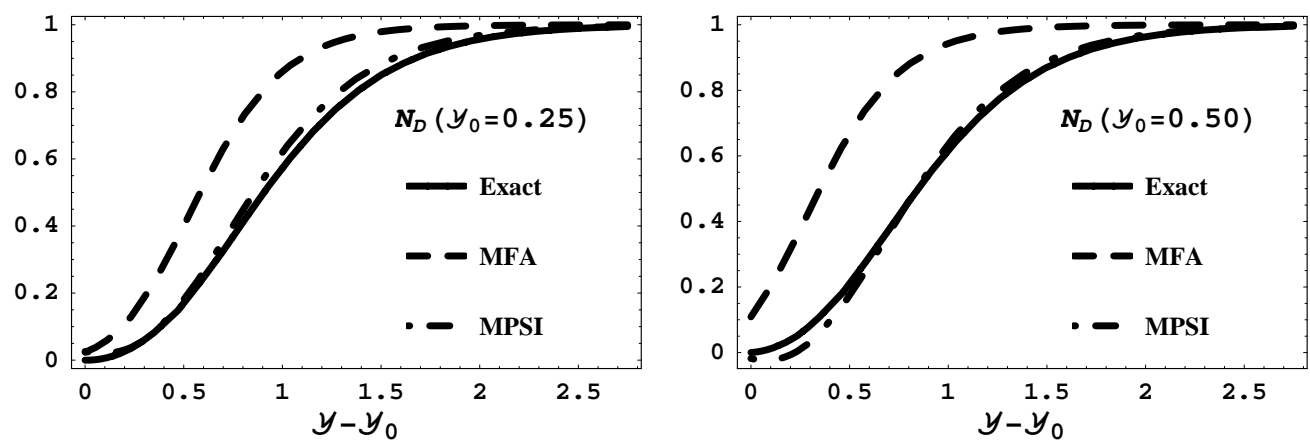

Figure 16: The comparison of the cross section of diffractive production with mass $\ln M^{2} \geq \mathcal{Y}_{0} /\left(\omega_{B F K L} \alpha_{S}^{2}\right)$ for the dipole-dipole scattering. The $x$-axis is $\mathcal{Y}-\mathcal{Y}_{0}=\omega_{B F K L} \alpha_{S}^{2}\left(Y-Y_{0}\right)=\left(4 \ln 2 \bar{\alpha}_{S} / \kappa\right)\left(Y-Y_{0}\right)$. The solid line is the exact solution while the dotted one shows the MFA result and dashed-dotted line shows the MPSI calculation.

diagrams gives us the new resulting Green's function $\left(G_{P}\right.$ of the Pomeron and we should try to build a new theory of the interacting Pomeron based on this function. It is easy to see that at $Y \rightarrow \infty$

$$
G_{P}(Y, u) \rightarrow \frac{1}{1-e^{-\kappa}}>1
$$

Therefore, the exchange of the resulting Pomeron leads (i) to the amplitude larger than unity but (ii) this amplitude is very close to the unitarity bound $\left(G_{P}=1\right)$. Therefore, we have a kind of black disc behaviour without the elastic cross section. We would lioke to recall that in the black disc limit $\sigma_{e l}=1 / 2 \sigma_{\text {tot }}$.

The fast decrease of the single diffractive production (see Fig. 9) shows that the effective triple Pomeron interaction vahishes at large $Y$. In other words, we can consider $G_{P}$ given by Eq. (6.1) as the exact solution of the multi interacting Pomeron system. On the other hand, the sum of enhanced diagrams gives the model for the two paricles irreducible diagrams in $s$-channel. To sum all diagrams we need to take into account also all two particle reducible diagrams. This sum is given by the eikonal (Glauber) formula, namely,

$$
A_{\text {asymp }} \rightarrow 1-e^{-G_{P}}=1-\exp \left(-\frac{1}{1-e^{-\kappa}}\right)<1
$$

Therefore we obtain a grey disc limit. The eikonalization gives a simple solution for all observables calculated here. However, Eq. (6.2) deserves a more careful consideration, as was noticed in Ref. [50], before being used. Eq. (6.2) gives the behaviour of the scattering amplitude at low energies but at $u \rightarrow 0(\gamma \rightarrow 1)$. Therefore, solving the Pomeron calculus we reduce the problem of high energy scattering to the scattering at low energies while the last step: to find the scattering amplitude at $u \rightarrow 1$ remains open and, perhaps, eikonalization is one of possible solutions of this problem.

\section{Acknowledgments:}

We want to thank Asher Gotsman, Edmond Iancu, Uri Maor and Al Mueller for very useful discussions on the subject of this paper. 
This research was supported in part by the Israel Science Foundation, founded by the Israeli Academy of Science and Humanities and by BSF grant \# 20004019.

\section{References}

[1] V. A. Abramovsky, V. N. Gribov and O. V. Kancheli, Yad. Fiz. 18, 595 (1973) [Sov. J. Nucl. Phys. 18, 308 (1974)].

[2] K. G. Boreskov, A. B. Kaidalov, V. A. Khoze, A. D. Martin and M. G. Ryskin, Eur. Phys. J. C 44 (2005) 523 [arXiv:hep-ph/0506211].

[3] L. V. Gribov, E. M. Levin and M. G. Ryskin, Phys. Rep. 100, 1 (1983).

[4] A. H. Mueller and J. Qiu, Nucl. Phys.,427 B 268 (1986) .

[5] L. McLerran and R. Venugopalan, Phys. Rev. D 49,2233, 3352 (1994); D 50,2225 (1994); D 53,458 (1996); D 59,09400 (1999).

[6] E. A. Kuraev, L. N. Lipatov, and F. S. Fadin, Sov. Phys. JETP 45, 199 (1977); Ya. Ya. Balitsky and L. N. Lipatov, Sov. J. Nucl. Phys. 28, 22 (1978).

[7] J. Bartels, M. Braun and G. P. Vacca, Eur. Phys. J. C40, 419 (2005) [arXiv:hep-ph/0412218]; J. Bartels and C. Ewerz, JHEP 9909, 026 (1999) [arXiv:hep-ph/9908454]; J. Bartels and M. Wusthoff, Z. Phys. C66, 157 (1995); $\quad$ A. H. Mueller and B. Patel, Nucl. Phys. B425, 471 (1994) [arXiv:hep-ph/9403256]; J. Bartels, Z. Phys. C60, 471 (1993).

[8] M. A. Braun, Phys. Lett. B632 (2006) 297 [arXiv:hep-ph/0512057]; arXiv:hep-ph/0504002; Eur. Phys. J. C16, 337 (2000) [arXiv:hep-ph/0001268]; M. Braun, Eur. Phys. J. C6, 321 (1999) [arXiv:hep-ph/9706373]; M. A. Braun and G. P. Vacca, Eur. Phys. J. C6, 147 (1999) [arXiv:hep-ph/9711486].

[9] H. Navelet and R. Peschanski, Nucl. Phys. B634, 291 (2002) [arXiv:hep-ph/0201285]; Phys. Rev. Lett. 82, 137 (1999), [arXiv:hep-ph/9809474]; Nucl. Phys. B507, 353 (1997) [arXiv:hep-ph/9703238].

[10] J. Bartels, L. N. Lipatov and G. P. Vacca, Nucl. Phys. B706, 391 (2005) [arXiv:hep-ph/0404110].

[11] V. N. Gribov, Sov. Phys. JETP 26, 414 (1968) [Zh. Eksp. Teor. Fiz. 53, 654 (1967)].

[12] A. H. Mueller, Nucl. Phys. B415, 373 (1994); ibid B437, 107 (1995).

[13] E. Levin and M. Lublinsky, Nucl. Phys. A730, 191 (2004) [arXiv:hep-ph/0308279].

[14] E. Levin and M. Lublinsky, Phys. Lett. B607, 131 (2005) [arXiv:hep-ph/0411121].

[15] E. Levin and M. Lublinsky, Nucl. Phys. A763,172 (2005), arXiv:hep-ph/0501173.

[16] E. Levin, Nucl. Phys. A763, 140 (2005), arXiv:hep-ph/0502243.

[17] I. Balitsky, [arXiv:hep-ph/9509348]; Phys. Rev. D60, 014020 (1999) [arXiv:hep-ph/9812311] Y. V. Kovchegov, Phys. Rev. D60, 034008 (1999), [arXiv:hep-ph/9901281]. 
[18] J. Jalilian-Marian, A. Kovner, A. Leonidov and H. Weigert, Phys. Rev. D59, 014014 (1999), [arXiv:hep-ph/9706377]; Nucl. Phys. B504, 415 (1997), [arXiv:hep-ph/9701284]; J. Jalilian-Marian, A. Kovner and H. Weigert, Phys. Rev. D59, 014015 (1999), [arXiv:hep-ph/9709432]; A. Kovner, J. G. Milhano and H. Weigert, Phys. Rev. D62, 114005 (2000), [arXiv:hep-ph/0004014]; E. Iancu, A. Leonidov and L. D. McLerran, Phys. Lett. B510, 133 (2001); [arXiv:hep-ph/0102009]; Nucl. Phys. A692, 583 (2001), [arXiv:hep-ph/0011241]; E. Ferreiro, E. Iancu, A. Leonidov and L. McLerran, Nucl. Phys. A703, 489 (2002), [arXiv:hep-ph/0109115]; H. Weigert, Nucl. Phys. A703, 823 (2002), [arXiv:hep-ph/0004044].

[19] Y. V. Kovchegov and E. Levin, Nucl. Phys. B 577 (2000) 221 [arXiv:hep-ph/9911523].

[20] M. Hentschinski, H. Weigert and A. Schafer, "Extension of the color glass condensate approach to diffractive Phys. Rev. D 73, 051501 (2006) [arXiv:hep-ph/0509272].

[21] A. Kovner, M. Lublinsky and H. Weigert, arXiv:hep-ph/0608258.

[22] E. Iancu and D. N. Triantafyllopoulos, Nucl. Phys. A756, 419 (2005) [arXiv:hep-ph/0411405]; Phys. Lett. B610, 253 (2005) [arXiv:hep-ph/0501193].

[23] A. H. Mueller, A. I. Shoshi and S. M. H. Wong, Nucl. Phys. B715, 440 (2005) [arXiv:hep-ph/0501088].

[24] E. Iancu, G. Soyez and D. N. Triantafyllopoulos, arXiv:hep-ph/0510094.

[25] A. Kovner and M. Lublinsky, ArXiv:hep-ph/0510047; arXiv:hep-ph/0503155; Phys. Rev. Lett. 94, 181603 (2005) [arXiv:hep-ph/0502119]; JHEP 0503, 001 (2005) [arXiv:hep-ph/0502071]; Phys. Rev. D71, 085004 (2005) [arXiv:hep-ph/0501198]; "Odderon and seven Pomerons: QCD Reggeon field theory from JIMWLK evolution," arXiv:hep-ph/0512316.

[26] Y. Hatta, E. Iancu, L. McLerran and A. Stasto, Nucl. Phys. A762 (2005) 272 [arXiv:hep-ph/0505235]; arXiv:hep-ph/0504182.

[27] Y. Hatta, E. Iancu, C. Marquet, G. Soyez and D. N. Triantafyllopoulos, "Diffusive scaling and the high-energy limit of deep inelastic scattering in QCD at large $N_{c}$," arXiv:hep-ph/0601150.

[28] M. Kozlov and E. Levin, "Solution for the BFKL pomeron calculus in zero transverse dimensions," arXiv:hep-ph/0604039.

[29] P. Rembiesa and A. M. Stasto, Nucl. Phys. B725 (2005) 251 [arXiv:hep-ph/0503223].

[30] A. I. Shoshi and B. W. Xiao, "Pomeron loops in zero transverse dimensions," arXiv:hep-ph/0512206.

[31] A. H. Mueller and B. Patel, Nucl. Phys. B425, 471 (1994).

[32] A. H. Mueller and G. P. Salam, Nucl. Phys. B475, 293 (1996), [arXiv:hep-ph/9605302]; G. P. Salam, Nucl. Phys. B461, 512 (1996).

[33] E. Iancu and A. H. Mueller, Nucl. Phys. A730 (2004) 460, 494, [arXiv:hep-ph/0308315],[arXiv:hep-ph/0309276].

[34] M. Kozlov and E. Levin, Nucl. Phys. A739 (2004) 291 [arXiv:hep-ph/0401118].

[35] Y. V. Kovchegov, Phys. Rev. D 72 (2005) 094009 [arXiv:hep-ph/0508276].

[36] A. I. Shoshi and B. W. Xiao, "Diffractive dissociation including pomeron loops in zero transverse dimensions," arXiv:hep-ph/0605282. 
[37] V. A. Khoze, A. D. Martin and M. G. Ryskin, Eur. Phys. J. C24 (2002) 581 [arXiv:hep-ph/0203122]; Eur. Phys. J. C21 (2001) 521 [arXiv:hep-ph/0105145]; Eur. Phys. J. C14 (2000) 525 [arXiv:hep-ph/0002072];

[38] A. B. Kaidalov, V. A. Khoze, A. D. Martin and M. G. Ryskin, Eur. Phys. J. C21 (2001) 521 [arXiv:hep-ph/0105145].

[39] E. Gotsman, H. Kowalski, E. Levin, U. Maor and A. Prygarin, "Survival probability for diffractive di-jet production at the LHC," EPJ (in press) arXiv:hep-ph/0512254; E. Gotsman, E. Levin, U. Maor, E. Naftali and A. Prygarin, "Survival probability of large rapidity gaps," Proceedings HERA and the LHC: A Workshop on the Implications of HERA for LHC Physic, ed.A. De Roeck and H. Jung,CERN-2005-014, DESY-PROC-2005-01, p.221, December 2005 [ arXiv:hep-ph/0511060] and reference there in.

[40] J. Bartels, S. Bondarenko, K. Kutak and L. Motyka, Phys. Rev. D 73 (2006) 093004 [arXiv:hep-ph/0601128].

[41] V. A. Khoze, A. D. Martin and M. G. Ryskin, "On the role of hard rescattering in exclusive diffractive Higgs production," arXiv:hep-ph/0602247.

[42] J. Miller, "Survival probability for Higgs diffractive production in high density QCD" ( in preparation).

[43] E. Levin and M. Lublinsky, Phys. Lett. B 521 (2001) 233 [arXiv:hep-ph/0108265]; Eur. Phys. J. C 22 (2002) 647 [arXiv:hep-ph/0108239].

[44] S. Bondarenko, E. Gotsman, E. Levin and U. Maor, Nucl. Phys. A 683 (2001) 649 [arXiv:hep-ph/0001260].

[45] K. G. Boreskov, A. B. Kaidalov, V. A. Khoze, A. D. Martin and M. G. Ryskin, Eur. Phys. J. C 44 (2005) 523 [arXiv:hep-ph/0506211].

[46] K. G. Boreskov, "Probabilistic model of Reggeon field theory," arXiv:hep-ph/0112325 and reference therein.

[47] A. H. Mueller and A. I. Shoshi, Nucl. Phys. B 692 (2004) 175 [arXiv:hep-ph/0402193].

[48] Y. V. Kovchegov, Phys. Rev. D 72 (2005) 094009 [arXiv:hep-ph/0508276].

[49] I. Gradstein and I. Ryzhik, "Tables of Series, Products, and Integrals", Verlag MIR, Moskau,1981.

[50] S. Bondarenko, L. Motyka, A. H. Mueller, A. I. Shoshi and B. W. Xiao, "On the equivalence of Reggeon field theory in zero transverse dimensions and reaction-diffusion processes," arXiv:hep-ph/0609213. 